\title{
Meta-analysis of quantitative diffusion-weighted MR imaging in the differential diagnosis of breast lesions
}

\author{
Xin Chen ${ }^{1 *}$, Wen-ling Li', Yi-li Zhang ${ }^{2}$, Qian Wu ${ }^{3}$, You-min Guo ${ }^{2}$, Zhi-lan Bai
}

\begin{abstract}
Background: To determine, in a meta-analysis, the diagnostic performance of quantitative diffusion-weighted (DW) MR imaging in patients with breast lesions.

Methods: English and Chinese studies published prior to June 2009 to assess the diagnostic performance of quantitative DWI in patients with breast lesions were reviewed and summarized with reference to the inclusion and exclusion criteria. Methodological quality was assessed by using the quality assessment of diagnostic studies (QUADAS) instrument. Publication bias analysis was performed by using Comprehensive Meta-analysis version 2. Meta-Disc version 1.4 was used to describe primary results and explore homogeneity by Chi-square test and inconsistency index; to explore threshold effect by receiver operator characteristic (ROC) space and Spearman correlation coefficient; and to pool weighted sensitivity and specificity by fixed or random effect model. A summary ROC (sROC) curve was constructed to calculate the area under the curve (AUC).
\end{abstract}

Results: Of 65 eligible studies, 13 with 615 malignant and 349 benign lesions were included in the original metaanalysis, among which heterogeneity arising from factors other than threshold effect and publication bias was explored. Methodological quality was moderate. The pooled weighted sensitivity and specificity with corresponding 95\% confidence interval $(\mathrm{Cl})$ in one homogenous subgroup of studies using maximum $b=1000$ $\mathrm{s} / \mathrm{mm}^{2}$ were $0.84(0.80,0.87)$ and $0.84(0.79,0.88)$ respectively. AUC of sROC was 0.9085 . Sensitivity analysis demonstrated that the pooled estimates were stable and reliable.

Conclusions: Quantitative DWI has a higher specificity to differentiate between benign and malignant breast lesions compared to that of contrast-enhanced MRI. However, large scale randomized control trials (RCTs) are necessary to assess its clinical value because of disunified diffusion gradient factor $b$ and diagnosis threshold.

\section{Background}

Breast cancer is the most common cancer in women worldwide [1]. Despite the improvement in the detection of breast cancer with the widespread application of mammography and ultrasound, differentiation between benign and malignant breast lesions remains a difficult diagnosis problem, especially in dense fibroglandular breasts. Numerous studies have been performed to assess the diagnostic performance of contrast-enhanced magnetic resonance imaging (MRI) in breast lesions [2-5]. Based on the enhancement pattern of lesions and

\footnotetext{
*Correspondence: chen_x129@163.com

'Department of Radiology, Second Affiliated Hospital of Medical College of

Xi'an Jiaotong University, Xi'an, Shannxi, China

Full list of author information is available at the end of the article
}

morphologic changes, breast MRI offers an overall sensitivity of $90 \%$ and specificity of $72 \%$ in detecting breast lesions according to a published Meta-analysis [6]. Therefore, the classification of a lesion detected on MRI as benign or malignant remains a challenge [6,7].

One of the latest advancements in MRI technology is the application of diffusion-weighted imaging (DWI) to offer quantitative evaluation of apparent diffusion coefficient (ADC). Quantitative DWI is a powerful imaging tool which provides unique information related to the diffusion of water molecules in the tissue and allows estimation of cellularity and tissue structure [8]. Restricted water movement in tumors with high cellularity usually leads to higher signal intensity and smaller $\mathrm{ADC}$ value $[7,9]$. $\mathrm{ADC}$ is measured by acquiring the MR 
signal at least twice, typically with and without diffusion weighting by the following formula: ADC $=\left[\operatorname{In}\left(\mathrm{S}_{0} / \mathrm{S}_{\mathrm{b}}\right)\right] /$ $\mathrm{b}$, where $\mathrm{S}_{\mathrm{b}}$ and $\mathrm{S}_{0}$ are the signal intensities on the DW imaging with and without diffusion weighting, respectively [10]. Theoretically, the diffusion sensitivity is easily varied by changing the diffusion gradient factor known as the "b value" which is proportional to the gradient amplitude, the duration of the applied gradient and the time interval between the paired gradients [8].

The potential role of quantitative DWI in characterizing breast lesions has been reported [11-18]. However, the reported sensitivity and specificity of diagnosing malignant breast tumors ranged from $62.5 \%$ to $92.8 \%$ and $45.8 \%$ to $96.7 \%$, respectively. Comparison of the diagnostic performance of breast quantitative DWI among the studies may have been compromised by differences in the patient characteristics, MR imaging techniques, and diagnostic criteria for malignancy in the studies. In this study, we pool a number of DWI studies of the breast to evaluate the diagnostic performance of DWI in breast lesion characterization.

\section{Methods}

\section{Literature Search}

The MedLine and CNKI (China National Knowledge Infrastructure) search were performed (Li WL and Bai ZL; MAY 20, 2009) by using the terms "DiffusionWeighted Imaging [MeSH] or DWI" for the diagnostic test and "Breast Neoplasms [MeSH] or breast cancer or breast lesions or breast" for the clinical domain. Terms regarding diagnostic performance were excluded in our search criteria to minimize missing relevant studies. We limited our search to publications in English and Chinese languages, female subjects, search term presence in the title or abstract of the article and publication date no later than May 2009. The Cochrane library, Ovid, Elsevier and Springer databases were also searched ( $\mathrm{Li}$ WL; June 10, 2009) for research articles with the same criteria. Review articles, letters, comments, case reports and unpublished articles were excluded. Extensive crosschecking of the reference lists of all retrieved articles was performed to supplement the list of articles.

\section{Selection of Articles}

Inclusion criteria were: varied pathology within the dataset; total number of lesions $\geq 30$, with the number of both malignant and benign lesions each $\geq 10$; histopathologic analysis (performed at surgery and biopsy) and follow-up by ultrasound, mammography, or MRI used as the reference standards; Sufficient information were presented to calculate the true-positive (TP), falsepositive (FP), true-negative (TN) and false-negative (FN) values for per-lesion statistics; When same data or subsets of data were presented in more than one article, the article with the most details and/or most recently published was chosen.

Articles were selected by two steps according to the road map of diagnostic systematic reviews and guidelines [19]. First, articles were excluded after inclusion and exclusion criteria were applied to the titles and abstracts of the articles fulfilling the search criteria. We then determined the final studies included in the metaanalysis after applying the same inclusion and exclusion criteria to the remaining content of the articles.

Studies were excluded if results of different MR imaging series (such as contrast-enhanced imaging and DWI) were presented in combination and could not be differentiated for performance assessment of tests on only DWI series.

\section{Quality Assessment and Data Extraction}

Two observers (Chen X and Zhang YL) independently selected eligible articles and extracted relevant data about study characteristics and examination results from each article by using a standardized form. To resolve disagreement between reviewers, a third reviewer (Guo YM) assessed all involved items. The majority opinion was used for analysis.

Methodological quality of included studies was assessed independently by two observers using the quality assessment of diagnostic studies (QUADAS) instrument, a quality assessment tool specifically developed for systematic reviews of diagnostic accuracy studies $[20,21]$.

The author, study nation, year of publication, number and age of subjects, and diagnostic test characteristics including $b$ values, mean ADC values of malignant and benign lesions, and diagnostic threshold for malignant lesions were extracted from each study.

Values for TP, FP, TN, FN, sensitivity (SEN), specificity (SPE), accuracy (ACC), positive predictive value (PPV), negative predictive value (NPV), positive likelihood ratio (PLR) and negative likelihood ratio (NLR) results in the detection of malignant lesions were extracted. SEN, SPE, ACC, PPV, NPV, PLR and NLR were calculated by the following formulas:

$$
\begin{aligned}
& \text { SEN }=\mathrm{TP} /(\mathrm{TP}+\mathrm{FN}) \\
& \mathrm{SPE}=\mathrm{TN} /(\mathrm{FP}+\mathrm{TN}) \\
& \mathrm{ACC}=(\mathrm{TP}+\mathrm{TN}) /(\mathrm{TP}+\mathrm{FP}+\mathrm{TN}+\mathrm{FN}) \\
& \mathrm{PPV}=\mathrm{TP} /(\mathrm{TP}+\mathrm{FP}) \\
& \mathrm{NPV}=\mathrm{TN} /(\mathrm{FN}+\mathrm{TN}) \\
& \mathrm{PLR}=\mathrm{SEN} /(1-\mathrm{SPE}) \\
& \mathrm{NLR}=(1-\mathrm{SEN}) / \mathrm{SPE}
\end{aligned}
$$


All tabulated results for different readers (interobserver), for multiple observations per reader (intraobserver) and for multiple $b$ values or techniques were counted as separate data sets.

\section{Meta-Analysis Homogeneity test}

We used the $Q$ statistic of Chi-square value test and inconsistency index (I-squared, $I^{2}$ ) to estimate the heterogeneity of individual studies contributing to the pooled estimate. The homogeneity was to evaluate if the differences across the studies were greater than expected by chance alone. $P<0.05$ suggests presence of heterogeneity beyond what could be expected by chance alone. I-squared $\left(I^{2}\right)$ describes the percentage of total variation across studies due to heterogeneity rather than chance and was also used as a measure to quantify the amount of heterogeneity. $I^{2}>50 \%$ suggests heterogeneity [22].

\section{Threshold effect}

Different thresholds may be used in included studies to define a positive test result due to lack of standardization. Differential threshold effect may be the reason for detectable difference in sensitivities and specificities of test accuracy studies. Representation of accuracy estimates from each study in a receiver operating characteristic (ROC) space and computation of Spearman correlation coefficient between the log (SEN) and log (1-SPE) were assessed for threshold effect. A typical pattern of "shoulder arm" plot in a ROC space and a strong positive correlation would suggest threshold effect $[23,24]$.

\section{Publication bias analysis}

Studies with optimistic results are more likely to be submitted and accepted for publication than studies with unfavorable results. Since publication biases would tend to exaggerate clinical effects resulting in potentially erroneous clinical decision making, it is important to assess the likely extent of the bias and its potential impact on the conclusions [25]. Publication bias can be visually examined after construction of a funnel plot and quantitatively detected by fail-safe N (NFS). In the absence of publication bias, the data points form a symmetric funnel-shaped distribution, whereas an asymmetric distribution indicates the presence of publication bias [26]. NFS refers to the exact number of hypothetical studies with null results that would be required to nullify the statistical significance of combined effect [27]. A relatively small NFS should be cause for concern. However, if NFS is large, we can be confident the combined effect, while possibly inflated by the exclusion of some studies, is nevertheless not zero [28].

\section{Statistical pooling}

Statistical pooling is not always necessary in every systematic review of test accuracy studies. The necessary precondition for pooling estimates is that the studies and results are reasonably homogeneous. The estimates can be pooled by the fixed effect model (FEM) or by the random effect model (REM) to incorporate variation among studies, and the output can be presented graphically as forest plots. If heterogeneity due to threshold effect is present, the accuracy data can be pooled by fitting a summary ROC (sROC) curve and summarizing that curve by means of the area under the curve (AUC). A sROC curve summarizes and combines the true and false positive rates from different diagnostic studies. The overall performance of diagnostic studies can be visualized and reflected by a sROC curve without being affected by a change of threshold values [29]. The best diagnostic modality would yield a point in the upper left corner or coordinate $(0,1)$ of the sROC space, representing $100 \%$ sensitivity (no false negatives) and 100\% specificity (no false positives) at the individual subject level. Similarly, AUC ranges from 1 for a perfect test that always diagnoses correctly, to 0 for a test that never does so in single studies or meta-analyses. If there is heterogeneity due to sources other than threshold effect, pooling should only be attempted within homogeneous subsets [23].

\section{Sensitivity analysis}

The pooled estimates were reappraised when suspicious studies were excluded, and the reappraised results were compared with the original results to assess stability and reliability of our meta-analysis.

The homogeneity test, threshold effect analysis, pooled weighted sensitivity and specificity, sROC curve and sensitivity analysis were performed by using Meta-Disc version 1.4 [23]. Publication bias analysis was performed by using Comprehensive Meta-analysis version 2 [30].

\section{Results}

With the computer search and manual cross-checking of reference lists, 65 abstracts (26 English and 39 Chinese) were retrieved. After reading the titles and abstracts, we found 25 eligible articles (15 English and 10 Chinese). After reading the full texts, we excluded 12 of the 25 relevant articles for the following reasons: The objective of studies was not to explore the diagnostic performance of $1.5 \mathrm{~T}$ MR DWI on breast lesions [31-34] $(n=4)$; Benign breast lesions in the study were all fibroadenomas [35] $(n=1)$; Researchers did not report data that could be used to construct or calculate TP, FP, TN and FN results $[15,17,36]$ $(n=3)$; Researchers presented results from a combination of different MR series that could not be differentiated for assessment of single DWI [37] $(n=1)$; Data or subsets of data were presented in other articles $[38,39](n=2)$. Thirteen articles (5 English and 8 Chinese) fulfilled all inclusion and exclusion criteria and 
were selected for data extraction and data analysis [11,13,14,16,18,40-47].

Nine hundred and sixty four breast lesions (615 malignant, 349 benign) were included from 13 studies. The mean ADC values of malignancy ranged from 0.87 to $1.36 \times 10^{-3} \mathrm{~mm}^{2} / \mathrm{s}$. The mean ADC values of benign lesions ranged from 1.00 to $1.82 \times 10^{-3} \mathrm{~mm}^{2} / \mathrm{s}$. The cutoff values differentiating malignant and benign lesions ranged from 0.90 to $1.76 \times 10^{-3} \mathrm{~mm}^{2} / \mathrm{s}$ while the sensitivity and specificity ranged from $63 \%$ to $100 \%$ and $46 \%$ to $97 \%$, respectively. The abstracted data of these individual studies are summarized in Tables 1 and 2. Two or multiple subsets of data in one study were included because: The two methods of echo planar imaging (EPI) and half-Fourier acquired single-shot turbo spin echo (HASTE) were used to perform breast DWI in Baltzer et al. [40]; Marini et al. used $1.1 \times 10^{-3} \mathrm{~mm}^{2} / \mathrm{s}$ (mean diffusivity of benign lesions - $1 \mathrm{SD}$ ) and $1.31 \times 10^{-3}$ $\mathrm{mm}^{2} / \mathrm{s}$ (mean diffusivity of malignant lesions $-2 \mathrm{SD}$ ) as cutoff between benign and malignant breast lesions [18]; $\mathrm{ADC}$ values were measured by two radiologists independently in Rubesova et al. [14]; Multiple b values were used in some studies $[41,42,44,45]$.

Results of distribution of study design characteristics in 13 studies according to QUADAS items are shown in Table 3. Most studies have a suboptimal design in regard to the reporting of selection criteria $(67.2 \%$ for "yes" responses to question 2), the description of the execution of the reference standard $(76.9 \%$ for "no" responses to question 9), the interpretation of the reference standard results without knowledge of the index test results and the interpretation of the index test results without knowledge of the reference standard (84.6\% and $100 \%$ for "unclear" responses to question 10 and 11 respectively), reporting of uninterpretable and/or intermediate test results $(69.2 \%$ for "no" responses to question 13) and explanation of withdrawals from the study (76.9\% for "no" responses to question 14).

The forest plots of sensitivities and specificities from 23 subsets of data from all 13 studies are shown in Figure 1. A homogeneity test of sensitivity and specificity shows $Q=58.20(P<0.0001), I^{2}=62.2 \%$ and $Q=61.44(P<0.0001), I^{2}=64.2 \%$, respectively. Therefore, notable heterogeneities are detected. The next step is the representation of sensitivity against 1 -specificity from each study in a ROC space to explore the threshold effect. The pattern of the points in this plot is not a "shoulder-arm" shape (Figure 2). A Spearman rank correlation is performed as a further test for threshold effect. The Spearman correlation coefficient was equal to $0.097(P=0.66)$ and indicates that there should be other factors than threshold effect resulting in variations in accuracy estimates among individual studies.

The funnel plot in Figure 3 shows that the studies are distributed symmetrically about the combined effect size and yield a z-value of 4.46191 with corresponding $P$-value of 0.00001 . The NFS is 92 . This means that we would need to locate and include 92 "null" studies in order for the $P$ to exceed 0.05 . There is no significant publication bias, and actual combined effect size is equal to the theoretical combined effect size.

Having found notable heterogeneity beyond threshold effect and publication bias, we then focus on the subgroup of studies using maximum $b=1000 \mathrm{~s} / \mathrm{mm}^{2}$. A homogeneity test of sensitivity and specificity shows $Q=16.39(P=0.1271), I^{2}=32.9 \%$ and $Q=15.81(P=$ $0.1483), I^{2}=30.4 \%$ respectively. The pooled weighted sensitivity and specificity with corresponding 95\% CIs

Table 1 The characteristics of included studies

\begin{tabular}{|c|c|c|c|c|c|c|c|}
\hline Author & $\begin{array}{c}\text { Publication } \\
\text { Year }\end{array}$ & Country & $\begin{array}{c}\text { Mean (range) age } \\
\text { (years) }\end{array}$ & $\begin{array}{c}\text { No. of } \\
\text { patients }\end{array}$ & $\begin{array}{l}\text { No. of total } \\
\text { lesions }\end{array}$ & $\begin{array}{l}\text { No. of benign } \\
\text { lesions }\end{array}$ & $\begin{array}{l}\text { No. of malignant } \\
\text { lesions }\end{array}$ \\
\hline Baltzer(40) & 2009 & Germany & $54(\mathrm{~N})$ & 65 & 74 & 35 & 39 \\
\hline Marini(18) & 2007 & Italy & 53 (24-79) & 60 & 63 & 21 & 42 \\
\hline $\begin{array}{l}\text { Rubesova } \\
\text { (14) }\end{array}$ & 2006 & Belgium & $52(25-74)$ & 78 & 87 & 22 & 65 \\
\hline $\begin{array}{l}\text { Woodhams } \\
\text { (13) }\end{array}$ & 2005 & Japan & $53(14-88)$ & 190 & 191 & 24 & 167 \\
\hline Guo(11) & 2002 & China & $58(25-75)$ & 52 & 55 & 24 & 31 \\
\hline Huang(41) & 2008 & China & $50(31-77)$ & $\mathrm{N}$ & 56 & 24 & 32 \\
\hline $\operatorname{Jin}(42)$ & 2008 & China & $N(N)$ & 56 & 60 & 20 & 40 \\
\hline Tang(43) & 2008 & China & $51(33-76)$ & 48 & 70 & 25 & 45 \\
\hline $\mathrm{Gu}(44)$ & 2008 & China & $51(33-83)$ & 83 & 95 & 52 & 43 \\
\hline $\operatorname{Lou}(45)$ & 2007 & China & $42(18-71)$ & 50 & 58 & 26 & 32 \\
\hline Luo(16) & 2007 & China & $43(24-65)$ & 52 & 60 & 33 & 27 \\
\hline $\operatorname{Li}(46)$ & 2005 & China & $45(24-68)$ & 35 & 41 & 13 & 28 \\
\hline Zhao(47) & 2005 & China & $43(21-72)$ & 46 & 54 & 30 & 24 \\
\hline
\end{tabular}

Note: $\mathrm{N}=$ not mentioned 
Table 2 The ADC measurement of included studies $(\times 10-3 \mathrm{~mm} 2 / \mathrm{s})(\bar{x} \pm S D)$.

\begin{tabular}{|c|c|c|c|c|c|}
\hline Author & B value $\left(\mathrm{s} / \mathrm{mm}^{2}\right)$ & Mean ADC of malignant & Mean ADC of benign & Mean ADC of normal & Threshold \\
\hline \multirow[t]{2}{*}{ Baltzer } & $0,750,1000$ & $1.05 \pm 0.33$ & $1.63 \pm 0.42$ & $\mathrm{~N}$ & 1.23 \\
\hline & 0,800 & $1.09 \pm 0.38$ & $1.67 \pm 0.40$ & N & 1.24 \\
\hline \multirow[t]{2}{*}{ Marini } & 0,1000 & $0.95 \pm 0.18$ & $1.48 \pm 0.37$ & $N$ & 1.10 \\
\hline & & & & & 1.31 \\
\hline \multirow[t]{2}{*}{ Rubesova } & $0,200,400,600,800,1000$ & $0.95 \pm 0.02$ & $1.51 \pm 0.07$ & N & 1.15 \\
\hline & & $0.99 \pm 0.03$ & $1.47 \pm 0.08$ & N & 1.10 \\
\hline Woodhams & 0,750 & $1.22 \pm 0.31$ & $1.67 \pm 0.54$ & $2.09 \pm 0.27$ & 1.60 \\
\hline Guo & 0,1000 & $0.97 \pm 0.20$ & $1.57 \pm 0.23$ & N & 1.30 \\
\hline \multirow[t]{2}{*}{ Huang } & 0,500 & $1.02 \pm 0.18$ & $1.61 \pm 0.32$ & $1.67 \pm 0.21$ & 1.32 \\
\hline & 0,1000 & $0.99 \pm 0.16$ & $1.59 \pm 0.33$ & $1.65 \pm 0.21$ & 1.25 \\
\hline \multirow[t]{2}{*}{ Jin } & 0,600 & $1.33 \pm 0.36$ & $1.82 \pm 0.31$ & $2.05 \pm 0.33$ & 1.44 \\
\hline & 0,1000 & $1.08 \pm 0.32$ & $1.61 \pm 0.33$ & $1.85 \pm 0.33$ & 1.18 \\
\hline \multirow[t]{2}{*}{ Tang } & 0,800 & $1.15 \pm 0.19$ & $1.47 \pm 0.25$ & $N$ & $N$ \\
\hline & 0,1000 & $1.08 \pm 0.19$ & $1.42 \pm 0.26$ & $N$ & 1.38 \\
\hline \multirow[t]{3}{*}{ Gu } & 0,500 & $1.36 \pm 0.38$ & $1.64 \pm 0.34$ & $1.77 \pm 0.39$ & 1.5 \\
\hline & 0,1000 & $1.18 \pm 0.31$ & $1.39 \pm 0.32$ & $1.56 \pm 0.33$ & 1.3 \\
\hline & 0,2000 & $0.82 \pm 0.20$ & $1.00 \pm 0.23$ & $0.90 \pm 0.27$ & 0.90 \\
\hline \multirow[t]{4}{*}{ Lou } & 0,400 & $1.28 \pm 0.48$ & $1.71 \pm 0.42$ & $2.06 \pm 0.48$ & 1.76 \\
\hline & 0,600 & $1.18 \pm 0.41$ & $1.59 \pm 0.41$ & $1.92 \pm 0.53$ & 1.64 \\
\hline & 0,800 & $1.11 \pm 0.41$ & $1.70 \pm 0.34$ & $1.82 \pm 0.48$ & 1.52 \\
\hline & 0,1000 & $1.05 \pm 0.38$ & $1.55 \pm 0.35$ & $1.75 \pm 0.52$ & 1.43 \\
\hline Luo & 0,800 & $0.87 \pm 0.23$ & $1.59 \pm 0.26$ & $1.98 \pm 0.31$ & 1.22 \\
\hline $\mathrm{Li}$ & 0,1000 & $1.21 \pm 0.26$ & $1.49 \pm 0.43$ & $N$ & 1.42 \\
\hline Zhao & 0,1000 & $0.91 \pm 0.25$ & $1.58 \pm 0.22$ & $1.78 \pm 0.51$ & 1.01 \\
\hline
\end{tabular}

Note: $\mathrm{N}=$ not mentioned; $\mathrm{ADC}$ = apparent diffusion coefficient; $\mathrm{SD}=$ standard deviation.

are $0.84(0.80,0.87)$ and $0.84(0.79,0.88)$ (Figure 4$)$ respectively, and expressed as AUC of sROC curve is 0.9085 (Figure 5).

Heterogeneity, the pooled weighted sensitivity and specificity, and AUC are analyzed again when [46] and [47] were excluded respectively due to the seemingly differential sensitivity and specificity from others (Table 4). The pooled sensitivity and specificity is similar, and the corresponding 95\% CIs are predominantly overlapped with each other. The homogeneity is not reversed. The results of sensitivity analysis demonstrate that the pooled estimates are stable and reliable.

\section{Discussion}

In this meta-analysis, we calculate an overall sensitivity of 0.84 (95\% CI: $0.82,0.87)$ and specificity of $0.79(95 \%$ CI: $0.75,0.82$ ) from 13 studies fulfilling all inclusion and exclusion criteria. However, there is notable heterogeneity among individual studies. Therefore, it is critical to investigate the source of heterogeneity to determine the potential impact factors and to evaluate the appropriateness of statistical pooling of accuracy estimates from various studies.

Meta-Disc is performed to assess threshold effect from representation of accuracy estimates from each study in a ROC space, and Spearman correlation coefficient is computed between the log (SEN) and log (1-SPE). Lack of "shoulder-arm" shape of the points in the ROC space and mild Spearman correlation coefficient (0.097) indicate that there should be factors other than differences in cutoff points for malignancy causing variations in accuracy estimates across individual studies. Additionally, publication bias is an usual source of heterogeneity in meta-analysis. Publication biases might exaggerate clinical effects resulting in potentially erroneous clinical decision making. In systematic reviews or meta-analysis, a thorough literature search is crucial to identify all relevant studies. In this meta-analysis, the search of several electronic databases is supplemented by checking references of relevant studies in order to reduce publication bias [25]. Although only English and Chinese articles are searched, the funnel plot and NFS analysis indicate that there is no significant publication bias in our metaanalysis.

The parameters used in DWI sequences may affect the results of ADC calculation, and the diffusion gradient factor $b$ is one of important parameters [8]. The variability in $\mathrm{b}$ values across studies makes the ranges and thresholds of ADC values difficult to interpret. Because $\mathrm{b}$ values used in the included studies varied, we needed 
Table 3 Evaluation of quality of included studies using the QUADAS tool.

\begin{tabular}{|c|c|c|c|c|c|c|c|c|c|c|c|c|c|c|c|c|}
\hline & Baltzer & Marini & Rubesova & Woodhams & Guo & Huang & Jin & Tang & $\mathrm{Gu}$ & Lou & Luo & $\mathrm{Li}$ & Zhao & $\begin{array}{l}\text { Yes } \\
(\%)\end{array}$ & $\begin{array}{l}\text { No } \\
(\%)\end{array}$ & $\begin{array}{l}\text { Unclear } \\
\text { (\%) }\end{array}$ \\
\hline 1 Patient spectrum & Y & Y & $Y$ & Y & Y & Y & Y & Y & Y & Y & Y & $\mathrm{Y}$ & $Y$ & 100 & 0 & 0 \\
\hline $\begin{array}{l}2 \text { Reporting of selection } \\
\text { critetia }\end{array}$ & Y & Y & Y & Y & $N$ & $N$ & Y & Y & $\mathrm{N}$ & N & Y & Y & Y & 69.2 & 30.8 & 0 \\
\hline 3 Reference standard & Y & Y & Y & Y & Y & Y & Y & Y & Y & Y & Y & Y & Y & 100 & 0 & 0 \\
\hline $\begin{array}{l}4 \text { Absence of disease } \\
\text { progression bias }\end{array}$ & Y & Y & Y & Y & Y & Y & Y & Y & Y & Y & Y & Y & Y & 100 & 0 & 0 \\
\hline $\begin{array}{l}5 \text { Absence of partial } \\
\text { vertification bias }\end{array}$ & Y & Y & Y & Y & Y & Y & Y & Y & Y & Y & Y & Y & Y & 100 & 0 & 0 \\
\hline $\begin{array}{l}6 \text { Absence of differential } \\
\text { vertification bias }\end{array}$ & N & N & $Y$ & $Y$ & $N$ & $Y$ & $Y$ & $Y$ & $Y$ & Y & Y & $N$ & $Y$ & 69.2 & 30.8 & 0 \\
\hline $\begin{array}{l}7 \text { Absence of incorporation } \\
\text { bias }\end{array}$ & $Y$ & $Y$ & $Y$ & $Y$ & $Y$ & $Y$ & $Y$ & $Y$ & $Y$ & $Y$ & $Y$ & $Y$ & $Y$ & 100 & 0 & 0 \\
\hline $\begin{array}{l}8 \text { Description of index text } \\
\text { execution }\end{array}$ & $Y$ & $Y$ & $Y$ & $Y$ & $Y$ & $Y$ & $Y$ & $Y$ & $Y$ & $Y$ & $Y$ & $Y$ & $Y$ & 100 & 0 & 0 \\
\hline $\begin{array}{l}9 \text { Description of reference } \\
\text { standard execution }\end{array}$ & $Y$ & $N$ & $N$ & $N$ & Y & $N$ & $\mathrm{~N}$ & $N$ & $Y$ & N & $N$ & $N$ & $\mathrm{~N}$ & 23.1 & 76.9 & 0 \\
\hline $\begin{array}{l}10 \text { Absence of test review } \\
\text { bias }\end{array}$ & $Y$ & $U$ & $U$ & U & $U$ & $Y$ & U & U & $U$ & U & $U$ & U & U & 15.4 & 0 & 84.6 \\
\hline $\begin{array}{l}11 \text { Absence of diagostic } \\
\text { review bias }\end{array}$ & $U$ & $U$ & U & U & U & $U$ & U & U & $U$ & U & $U$ & U & $U$ & 0 & 0 & 100 \\
\hline $\begin{array}{l}12 \text { Absence of clinical review } \\
\text { bias }\end{array}$ & $Y$ & $Y$ & $Y$ & $Y$ & $Y$ & $Y$ & $Y$ & $Y$ & $Y$ & $Y$ & $Y$ & $Y$ & $Y$ & 100 & 0 & 0 \\
\hline $\begin{array}{l}13 \text { Reporting of } \\
\text { uninterpretable/intermediate } \\
\text { results }\end{array}$ & $N$ & $N$ & $Y$ & $\mathrm{~N}$ & Y & $N$ & $\mathrm{~N}$ & $Y$ & $N$ & N & Y & $N$ & $N$ & 30.8 & 69.2 & 0 \\
\hline 14 Withdrawal & Y & U & Y & U & Y & $U$ & U & U & U & U & U & U & U & 23.1 & 76.9 & 0 \\
\hline
\end{tabular}

Note: $\mathrm{Y}=$ yes; $\mathrm{N}=$ no; $\mathrm{U}=$ unclear; $\mathrm{QUADAS}$ = quality assessment of diagnostic studies.

to explore whether b values were the source of heterogeneity. Meta-analysis is performed again in the subgroup of studies using maximum $\mathrm{b}=1000 \mathrm{~s} / \mathrm{mm}^{2}$ because more studies used $\mathrm{b}$ at $1000 \mathrm{~s} / \mathrm{mm}^{2}$ than any other value from Table 2 . The results show that there is no significant heterogeneity, and the pooled sensitivity and specificity with $95 \%$ CIs are $0.84(0.80,0.87)$ and $0.84(0.79,0.88)$ respectively. The area under the curve

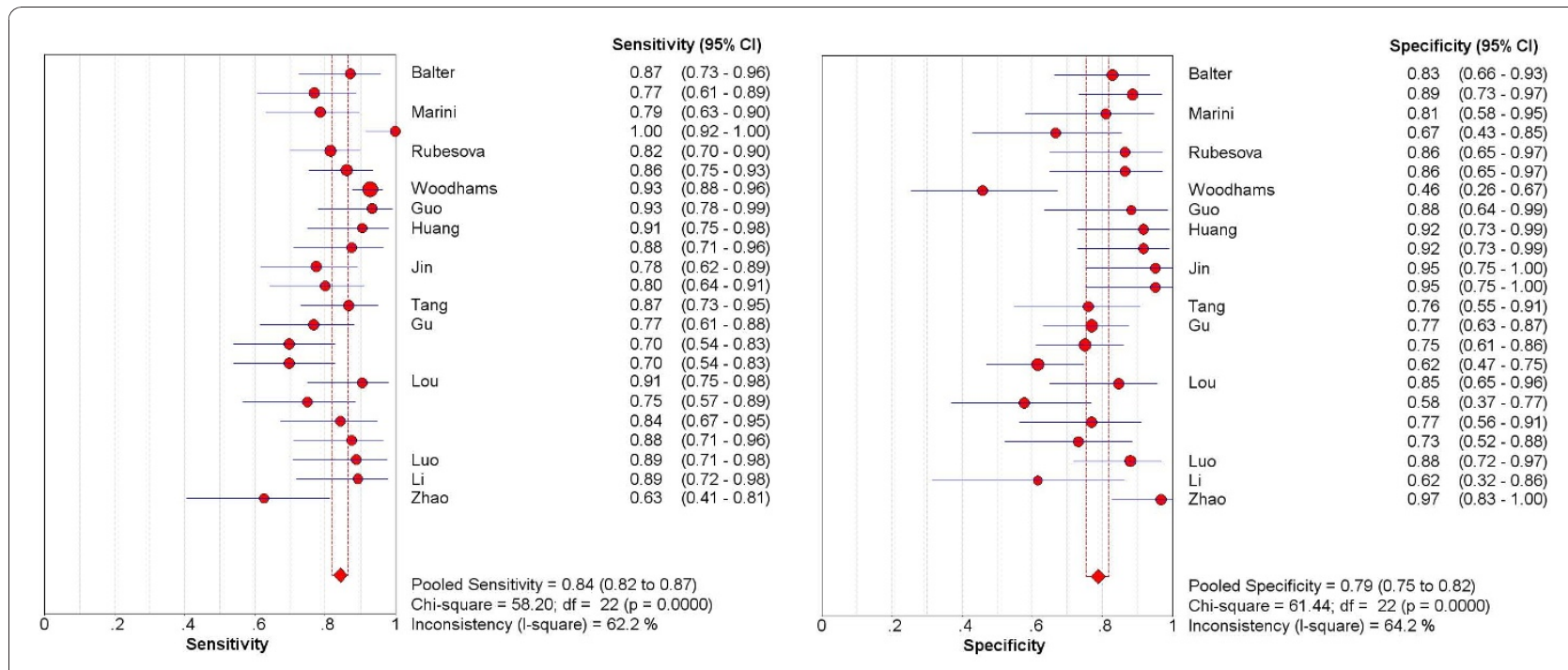

Figure 1 Forest plots of sensitivity and specificity, with corresponding $95 \% \mathrm{Cls}$ from all eligible studies. 


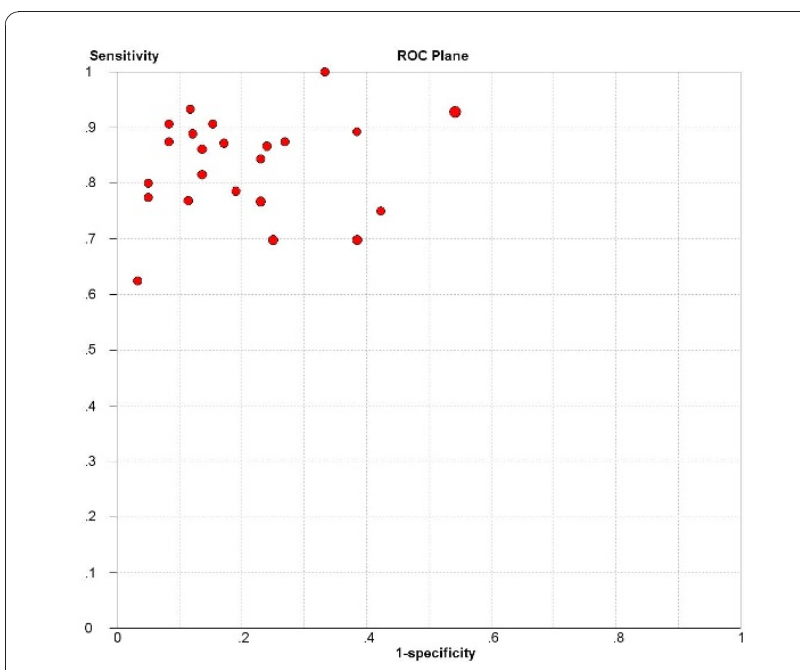

Figure 2 Sensitivity and 1-specficity plotted in receiver operating characteristic space for individual studies

of sROC is 0.9085 . Compared with the original results from all 13 eligible studies, the specificity is improved. However, we could not determine effect of other b values to pooled accuracy estimates because of the relatively small number of included studies. Furthermore, sensitivity analysis is performed in the subgroup of $b=1000 \mathrm{~s} / \mathrm{mm}^{2}$. When the studies with the maximum variance of sensitivity and specificity are excluded in sequence, the characteristics of heterogeneity are not changed and the variances of the pooled accuracy estimates are not significant. The sensitivity analysis shows that this meta-analysis in the subgroup is stable.

A previously published meta-analysis for contrastenhanced MRI including 44 studies reported the overall sensitivity of 0.90 (95\% CI: $0.88,0.92)$ and specificity of 0.72 (95\% CI: 0.67, 0.77) in patients with breast lesions

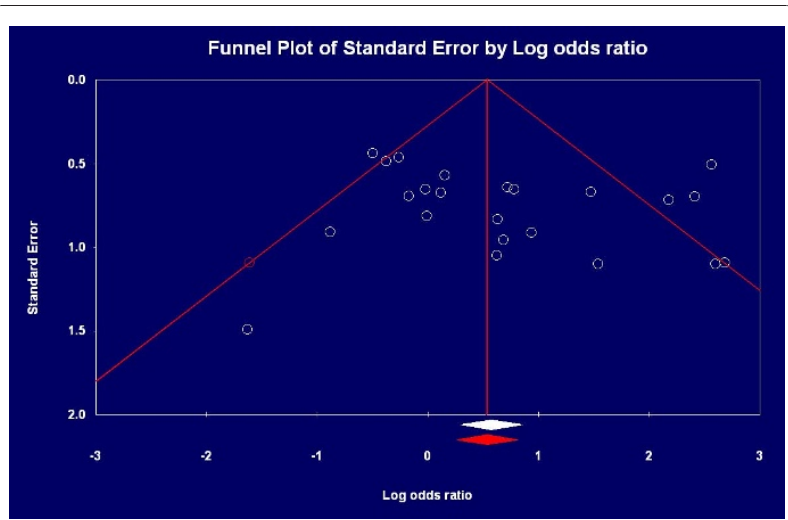

Figure 3 Funnel plot of eligible studies. Note: White flakes represent published articles, and red flake represents possibly missed article. White and red rhombuses represent actual and theoretical combined effect size respectively.
[6]. In comparison, our meta-analysis indicates that DWI has higher specificity but lower sensitivity. However, several differences and limitations in our meta-analysis should be noted. The significance of Peter et al. [6] lies in the diagnostic performance of MR imaging of small lesions detected at mammographic screening. Because a large proportion of these lesions are nonpalpable, they only include studies that enrolled at least one patient with nonpalpable lesion [6]. In our meta-analysis, we do not filter the studies using such criteria. In the included studies, Marini et al. selected lesions with diameter $>1 \mathrm{~cm}$ [18]. Rubesova et al. selected lesions with diameter $>0.7 \mathrm{~cm} \mathrm{[14]}$, and Tang et al. selected lesions with diameter $\leq 2 \mathrm{~cm}$ [43]. Lesion diameter ranged from 0.3 to $9 \mathrm{~cm}$ for other included studies. It is not certain that smaller lesions could be more easily missed in DW images since no included studies reported the number of nonpalpable lesions. Such variation might affect the overall estimates of the sensitivity and specificity. Because MR is one of the important methods for breast cancer diagnosing, DWI, having the advantages of a short examination time and no need to inject a contrast medium could be used in screening crowd to early detect nonpalpable lesions and to generate significant benefits to early tumor diagnosis and human health care needs in the future.

One limitation of our study is the suboptimal quality of included studies. Meta-analysis combines or integrates the results of several independent studies. The quality and reliability of a meta-analysis depends on the quality of included studies. We use the QUADAS tool for assessing methodological quality of individual studies. This tool was specifically developed for quality assessment of diagnostic accuracy studies included in systematic reviews and has been used to help identify severe methodological shortcomings [21]. Most included studies in this meta-analysis had a suboptimal design in regard to the reporting of selection criteria, the description of the execution of the reference standard, the interpretation of the reference standard results without knowledge of the index test results, the interpretation of the index test results without knowledge of the reference standard, reporting of uninterpretable and/or intermediate test results, or explanation of withdrawals from the study (Table 3).

In test accuracy studies, interpretation of the results of the index test may be influenced by knowledge of the results of the reference standard, and vice versa. This is known as review bias and may lead to inflated measures of diagnostic accuracy. As the index test, DWI was always performed first, and interpretation of the results of the DWI was usually done without knowledge of the results of the reference standard. However, if test result was interpreted at a later date, after both DWI and 


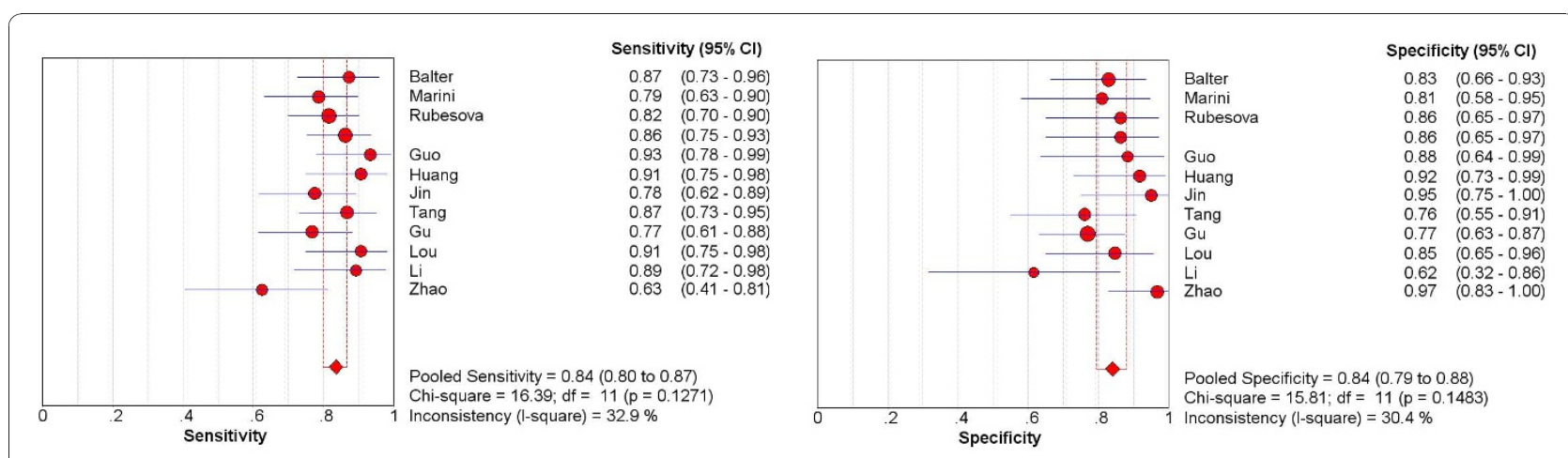

Figure 4 Forest plots of sensitivity and specificity, with corresponding $95 \% \mathrm{Cls}$ from studies using maximum b $=1000 \mathrm{~s} / \mathrm{mm}^{2}$

reference standard had been completed, then it was still important for a study to provide a description of whether the interpretation of each test was performed blind to the results of the other tests. Uninterpretable results produced in test accuracy studies are often not reported. Instead, they are simply removed from the analysis. This problem may lead to the biased assessment of the test characteristics. Whether bias will arise

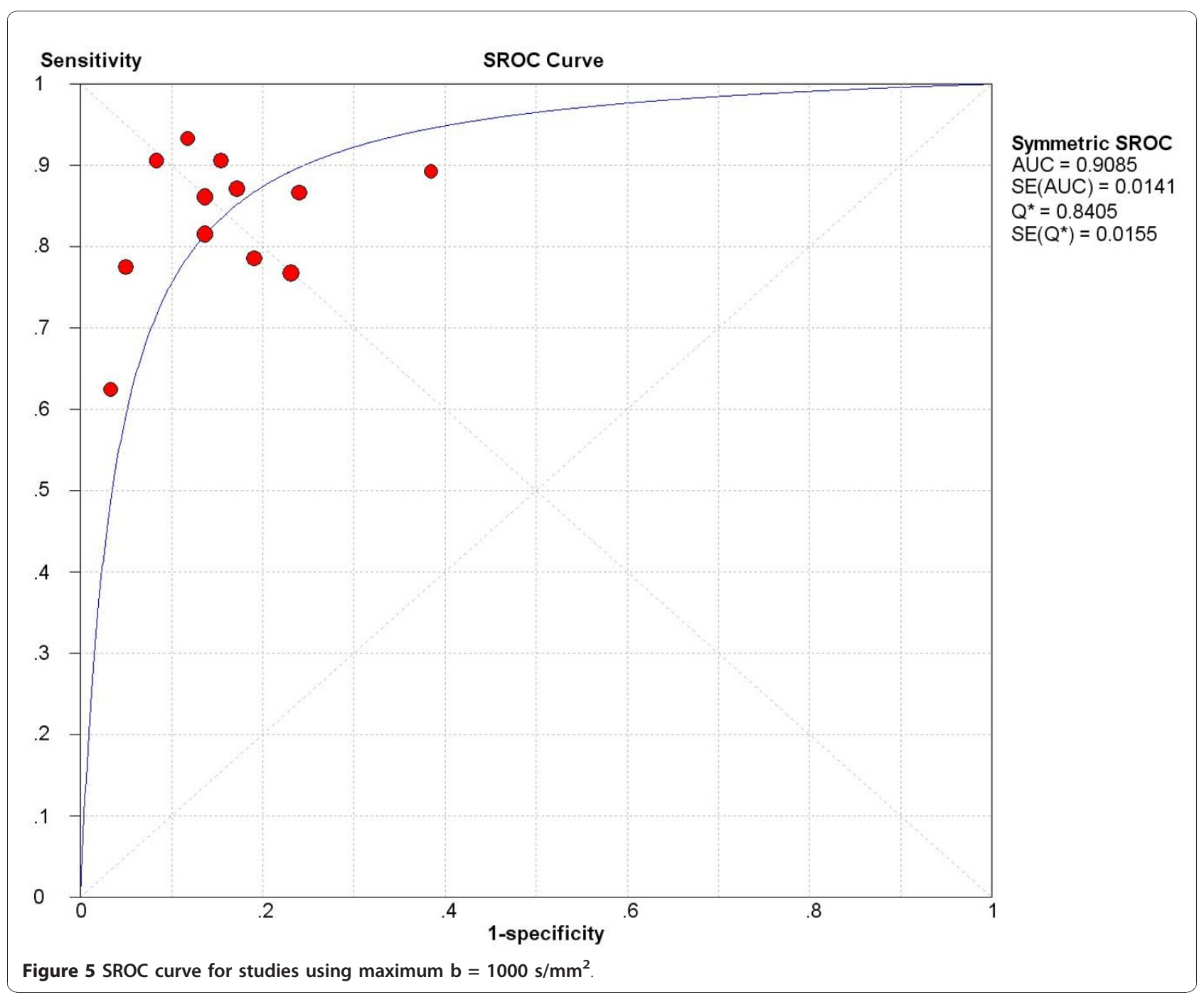


Table 4 Results of heterogeneity and the pooled estimations excluding suspicious studies.

\begin{tabular}{|c|c|c|c|c|c|c|c|}
\hline \multirow[t]{2}{*}{ Study } & \multirow{2}{*}{$\begin{array}{l}\text { Sensitivity } \\
(95 \% \mathrm{Cl})\end{array}$} & \multicolumn{2}{|c|}{ Heterogeneity } & \multirow{2}{*}{$\begin{array}{c}\text { Specificity } \\
(95 \% \mathrm{CI})\end{array}$} & \multicolumn{2}{|c|}{ Heterogeneity } & \multirow[t]{2}{*}{ AUC } \\
\hline & & $P$ & $I^{2}$ & & $P$ & $1^{2}$ & \\
\hline$b=1000$ & $0.84(0.80,0.87)$ & 0.1271 & $32.9 \%$ & $0.84(0.79,0.88)$ & 0.1483 & $30.4 \%$ & 0.9085 \\
\hline Excluding Li's & $0.83(0.79,0.86)$ & 0.1153 & $35.4 \%$ & $0.85(0.80,0.89)$ & 0.2999 & $15.1 \%$ & 0.9117 \\
\hline Excluding Zhao's & $0.85(0.80,0.87)$ & 0.4180 & $2.3 \%$ & $0.84(0.79,0.88)$ & 0.6377 & 0 & 0.9118 \\
\hline
\end{tabular}

Note: $\mathrm{AUC}=$ area under the curve; $\mathrm{Cl}=$ confidence interval.

depends on the possible correlation between uninterpretable test results and the true disease status. If uninterpretable results occur randomly and are not related to the true disease status of the individual, these should not have any effect on test performance. However, whatever the cause of uninterpretable results, it is important that they are reported so that the impact of these results on test performance can be determined. Patients' withdrawal from the study can occur prior to the results of either or both of the index test and reference standard in diagnostic studies being available. If patients lost to follow-up differ systematically from those who remain in studies, then estimates of test performance may be biased. Therefore, it is important to report patients lost to follow-up. In brief, most studies in evaluating the diagnostic values of DWI for breast lesions had some clinical operational challenges. Therefore a systematic data reporting method such as the standards for reporting diagnostic accuracy (STARD) should be advocated to improve the quality of reporting test accuracy studies [48].

All of individuals showed that the mean ADC value of malignant lesions was lower than that of benign lesions. However, the reported mean ADC values of malignant and benign tumors ranged from 0.87 to 1.36 and 1.00 to $1.82 \times 10^{-3} \mathrm{~mm}^{2} / \mathrm{s}$, respectively, resulting in recommended threshold values of ADC ranging from 0.90 to $1.76 \times 10^{-3} \mathrm{~mm}^{2} / \mathrm{s}$. Even in the subgroup of studies using maximum $\mathrm{b}=1000 \mathrm{~s} / \mathrm{mm}^{2}$, the minimum and maximum threshold values were $1.10 \times 10^{-3} \mathrm{~mm}^{2} / \mathrm{s}$ $[14,18]$ and $1.38 \times 10^{-3} \mathrm{~mm}^{2} / \mathrm{s}$ [43], respectively. The substantial variance of threshold values might be influenced by different $b$ values, selection method, bias of patient selection, pathological characteristic of lesions and measurement of ADC values. This meta-analysis does not seem to predict or determine the unified threshold value to differentiate malignant and benign breast lesions because selection of the threshold value should be determined according to the purpose of examination. For example, a relatively higher threshold value may be recommended to minimize missing malignancy in breast cancer screening. If DWI is appended to the contrast-enhanced MRI, a relatively lower threshold value may be recommended to reduce false positive results.

\section{Conclusion}

ADC measurement of DWI is useful for differentiation between malignant and benign breast lesions with pooled sensitivity of 0.84 and specificity of 0.84 in one homogenous subgroup of studies using maximum $\mathrm{b}=1000 \mathrm{~s} / \mathrm{mm}^{2}$, and the area under curve of sROC was 0.9085 . DWI has a higher specificity but lower sensitivity compared to that of contrast-enhanced MRI. However, with all of methodological issues considered, results must be interpreted with caution. Large scale randomized control trials (RCTs) are necessary to assess and confirm its clinical value. A threshold value for malignant/benign lesions classification could not be made based on this study because it is influenced by different $b$ values, bias of patient selection, lesions' pathological characteristics and ADC measurement. Selection of the threshold value should be determined according to the purpose of examination.

\section{Abbreviations}

DWI: Diffusion-Weighted Imaging; sROC: Summary Receiver Operating Characteristic; ADC: Apparent Diffusion Coefficient; MRI: Magnetic Resonance Imaging; TP: True Positive; FP: False Positive; TN: True Negative; FN: False Negative; SEN: Sensitivity; SPE: Specificity; ACC: Accuracy; PPV: Positive Predictive Value; NPV: Negative Predictive Value; PLR: Positive Likelihood Radio; NLR: Negative Likelihood Ratio; $P^{2}$ : Inconsistency Index; NFS: Fail-Safe N; FEM: Fixed effect Mode; REM: Random Effect Mode; DOR: Diagnostic Odds Ratio; AUC: Area Under the Curve; 95\%Cl: 95\% Confidence Interval; RCT: Randomized Control Trial.

\section{Acknowledgements}

This study was supported by National Natural Science Foundation of China (No.30701008).

\section{Author details}

1Department of Radiology, Second Affiliated Hospital of Medical College of Xi'an Jiaotong University, Xi'an, Shannxi, China. ${ }^{2}$ Department of Radiology, First Affiliated Hospital of Medical College of Xi'an Jiaotong University, Xi'an, Shannxi, China. ${ }^{3}$ Department of Epidemiology, Medical College of Xi'an Jiaotong University, Xi'an, Shannxi, China.

\section{Authors' contributions}

Chen X conceived of the study concept and participated in its design, quality assessment, statistical analysis, manuscript drafting and editing and approval for important intellectual concepts. Li WL and Bai ZL participated in the literature research, manuscript drafting and editing. Zhang YL participated in design and quality assessment. Wu Q participated in data acquisition and statistical analysis. Gou YM conceived of the study concept and participated in design, manuscript drafting and editing, data analysis 
and interpretation and approval for important intellectual concepts. All authors read and approved the final manuscript.

\section{Competing interests}

The authors declare that they have no competing interests.

Received: 21 January 2010 Accepted: 29 December 2010 Published: 29 December 2010

\section{References}

1. Parkin DM, Bray F, Ferlay J, Pisani P: Global cancer statistics, 2002. CA Cancer J Clin 2005, 55:74-108

2. Boetes C, Veltman J, van Die L, Bult P, Wobbes T, Barentsz JO: The role of MRI in invasive lobular carcinoma. Breast Cancer Res Treat 2004, 86:31-37.

3. Kriege $M$, Brekelmans $C T$, Boetes $C$, Besnard PE, Zonderland HM, Obdeijn IM, Manoliu RA, Kok T, Peterse H, Tilanus-Linthorst MM, Muller SH, Meijer S, Oosterwijk JC, Beex LV, Tollenaar RA, de Koning HJ, Rutgers EJ, Klijn JG, Magnetic Resonance Imaging Screening Study Group: Efficacy of MRI and mammography for breast-cancer screening in women with a familial or genetic predisposition. N Engl J Med 2004, 351:427-437.

4. Schnall MD, Blume J, Bluemke DA, DeAngelis GA, DeBruhl N, Harms S, Heywang-Köbrunner SH, Hylton N, Kuhl CK, Pisano ED, Causer P, Schnitt SJ, Thickman D, Stelling CB, Weatherall PT, Lehman C, Gatsonis CA: Diagnostic architectural and dynamic features at breast MR imaging: multicenter study. Radiology 2006, 238:42-53.

5. Van Goethem M, Tjalma W, Schelfout K, Verslegers I, Biltjes I, Parizel P: Magnetic resonance imaging in breast cancer. Eur J Surg Oncol 2006, 32:901-910.

6. Peters NHGM, Borel Rinkes IHM, Zuithoff NPA, Mali WPTM, Moons KGM, Peeters PHM: Meta-analysis of MR imaging in the diagnosis of breast lesions. Radiology 2008, 246:116-124.

7. Kuhl CK: Concepts for differential diagnosis in breast MR imaging. Magn Reson Imaging Clin N Am 2006, 14:305-328.

8. Koh DM, Collins DJ: Diffusion-weighted MRI in the body: application and challenges in oncology. Am J Roentgenol 2007, 188:1622-1635.

9. Yoshikawa MI, Ohsumi S, Sugata S, Kataoka M, Takashima S, Mochizuki T, Ikura $\mathrm{H}$, Imai Y: Relation between cancer cellularity and apparent diffusion coefficient values using diffusion-weighted magnetic resonance imaging in breast cancer. Radiat Med 2008, 26:222-226.

10. Herneth AM, Guccione S, Bednarski M: Apparent diffusion coefficient: a quantitative parameter for in vivo tumor characterization. Eur J Radiol 2003, 45:208-213.

11. Guo Y, Cai YQ, Cai ZL, Gao YG, An NY, Ma L, Mahankali S, Gao J: Differentiation of clinically benign and malignant breast lesions using diffusion-weighted imaging. J Magn Reson Imaging 2002, 16:172-178.

12. Sinha S, Lucas-Quesada FA, Sinha U, Debruhl N, Bassett LW: In vivo diffusion-weighted MRI of the breast: potential for lesion characterization. J Magn Reson Imaging 2002, 15:693-704.

13. Woodhams R, Matsunaga K, Kan S, Hata H, Ozaki M, Iwabuchi K, Kuranami M, Watanabe M, Hayakawa K: ADC mapping of benign and malignant breast tumors. Magn Reson Med Sci 2005, 4:35-42.

14. Rubesova E, Grell AS, De Maertelaer V, Metens T, Chao SL, Lemort M: Quantitative diffusion imaging in breast cancer: a clinical prospective study. J Magn Reson Imaging 2006, 24:319-324.

15. Kuroki Y, Nasu K, Kuroki S, Murakami K, Hayashi T, Sekiquchi R, Nawabo S: Diffusion-weighted imaging of breast cancer with the sensitivity encoding technique: analysis of the apparent diffusion coefficient value. Magn Reson Med Sci 2004, 3:79-85.

16. Luo J, Liu Y, Zhang L, Shi L: Application of diffusion weighted magnetic resonance imaging to differential diagnosis of breast diseases. Ai Zheng 2007, 26:168-171.

17. Park MJ, Cha ES, Kang BJ, Ihn YK, Jun HB: The role of diffusion-weighted imaging and the apparent diffusion coefficient (ADC) values for breast tumors. Korean J Radiol 2007, 8:390-396.

18. Marini C, lacconi C, Giannelli M, Cilotti A, Moretti M, Bartolozzi C: Quantitative diffusion-weighted MR imaging in the differential diagnosis of breast lesion. Eur Radiol 2007, 17:2646-2655.

19. Diagnostic Systematic Reviews: A Road Map (Version 3). [http://www. medepi.net/meta/guidelines/Diagnostic_Systematic_Reviews_Road_Map_V3. pdf.].
20. Whiting P, Rutjes AW, Reitsma JB, Bossuyt PM, Kleijnen J: The development of QUADAS: a tool for the quality assessment of studies of diagnostic accuracy included in systematic reviews. BMC Med Res Methodol 2003, 3:25.

21. Whiting P, Weswood ME, Rutjes AW, Reitsma JB, Bossuyt PN, Kleijnen J: Evaluation of QUADAS, A Tool for the Quality Assessment of Diagnostic Accuracy Studies (update). BMC Med Res Methodol 2006, 6:9.

22. Higgins JPT, Thompson SG, Deeks JJ, Altman DG: Measuring inconsistency in meta-analyses. BMJ 2003, 327:557-560.

23. Zamora J, Abraira V, Muriel A, Khan KS, Coomarasamy A: Meta-DiSc: a software for meta-analysis of test accuracy data. BMC Med Res Methodol 2006, 6:31.

24. Moses LE, Shapiro D, Littenberg B: Combining independent studies of a diagnostic test into a summary ROC curve: data-analytic approaches and some additional considerations. Stat Med 1993, 12:1293-1316.

25. Song F, Khan KS, Dinnes J, Sutton AJ: Asymmetric funnel plots and publication bias in meta-analyses of diagnostic accuracy. Int J Epidemiol 2002, 31:88-95.

26. Song F, Eastwood A, Gilbody S, Duley L, Sutton AJ: Publication and related biases. Health Technol Assess 2000, 4:10.

27. Orwin R: A fail-safe $\mathbf{N}$ for effect size in Meta-analysis. J Educ Behav Stat 1983, 8:157-159.

28. Persaud R: Misleading Meta-analysis: "Fail-safe N" is a useful mathematical measure of the stability of results. BMJ 1996, 312:125.

29. Deeks JJ: Systematic reviews in health care: Systematic reviews of evaluations of diagnostic and screening tests. BMJ 2001, 323:157-162.

30. Comprehensive Meta-analysis version 2. [http://www.meta-analysis.com/ index.html].

31. Zhang YL, Huang X, Du H, Zhang Y, Chen X, Wang P, Guo Y: The value of diffusion-weighted imaging in assessing the ADC changes of tissues adjacent to breast carcinoma. BMC Cancer 2009, 9:18.

32. Matsuoka A, Minato M, Harada M, Kubo H, Bandou Y, Tangoku A, Nakano K, Nishitani H: Comparison of 3.0-and 1.5-tesla diffusion-weighted imaging in the visibility of breast cancer. Radiat Med 2008, 26:15-20.

33. Yoshikawa MI, Ohsumi S, Sugata S, Kataoka M, Takashima S, Mochizuki T, Ikura H, Imai Y: Relation between cancer cellularity and apparent diffusion coefficient values using diffusion-weighted magnetic resonance imaging in breast cancer. Radiat Med 2008, 26:222-6.

34. Partridge SC, McKinnon GC, Henry RG, Hylton NM: Menstrual cycle variation of apparent diffusion coefficients measured in the normal breast using MRI. J Magn Reson Imaging 2001, 14:433-8.

35. Hatakenaka M, Soeda H, Yabuuchi H, Matsuo Y, Kamitani T, Oda Y, Honda MTsuneyoshi: Apparent diffusion coefficients of breast tumors: clinical application. Magn Reson Med Sci 2008, 7:23-29.

36. Feng $Y$, Liu S, Wang C, Tao X, Wang J, Wang J: Application of functional MRI in breast diseases. Chin J Radiol 2007, 41:457-462.

37. Yabuuchi H, Matsuo Y, Okafuji T, Kamitani T, Soeda H, Setoguchi T, Sakai S, Hatakenaka M, Kubo M, Sadanaga N, Yamamoto H, Honda H: Enhanced mass on contrast-enhanced breast MR imaging: Lesion characterization using combination of dynamic contrast-enhanced and diffusionweighted MR images. J Magn Reson Imaging 2008, 28:1157-65.

38. Woodhams R, Matsunaga K, Iwabuchi K, Kan S, Hata H, Kuranami M, Watanabe M, Hayakawa K: Diffusion-weighted imaging of malignant breast tumors: the usefulness of apparent diffusion coefficient (ADC) value and $A D C$ map for the detection of malignant breast tumors and evaluation of cancer extension. J Comput Assist Tomogr 2005, 29:644-649.

39. Guo Y, Cai Z, Cai Y, Ning A, Gao Y, Ma L, Liang Y: Differentiation of benign from malignant breast lesions by apparent diffusion coefficients. Chin J Radiol 2001, 35:132-135.

40. Balzer PAT, Renz DM, Herrmenn KH, Krumbein I, Gajda M, Camara O, Reichenbach JR, Kaiser WA: Diffusion-weighted imaging (DWI) in MR mammography (MRM): clinical comparison of echo planar imaging (EPI) and half-Fourier single-shot turbo spin echo (HASTE) diffusion techniques. Eur Radiol 2009, 19:1612-1620

41. Huang $X, D u H$, Zhang $Y$, Zhang $Y$ : The value of MR diffusion-weighted imaging in diagnosing the benign and malignant breast lesions. J Pract Radiol 2008, 24:526-533.

42. Jin G, Cai Y, An N, Li X, Liu M, Wang J: Clinical application of diffusionweighted imaging with ASSET technique for breast lesions. Clinical Radiology 2008, 27:796-799. 
43. Tang J, Yan F, Zhou M, Ye F, Xu P: Comparative study of diffusion weighted imaging and dynamic contrast enhancement MRI for the detection of small breast cancers. Chin J Radiol 2008, 42:152-156.

44. Gu Y, Fen X, Tang F, Peng W, Mao J, Yang W: Diffusion-weighted MRI in the breast: lesion characterization and parameter selection. Chin J Radiol 2007, 41:451-456.

45. Lou L, Zhang J, Shi G: The application of MR diffusion weighted imaging in differentiation of benign from malignant breast lesions. Clinical Radiology 2007, 26:350-353.

46. Li J, Zhang X, Cao K, Sun Y, Shan J, OuYang T: Clinical evaluation of combining dynamic contrast-enhanced MR imaging and diffusionweighted MR imaging for diagnosis of breast lesion. Chinese Journal of Medical Imaging technology 2005, 21:1821-1825.

47. Zhao B, Cai SF, Gao PH, Peng HJ: The research on distinguishing benign from malignant breast lesions by diffusion-weighted MR imaging. Chin J Radiol 2005, 39:497-500.

48. Bossuyt P, Reitsma J, Bruns D, Gatsonis CA, Glasziou PP, Irwig LM, Moher D, Rennie D, Vet HCW, Lijmer JG: The STARD statement for reporting studies of diagnostic accuracy: explanation and elaboration. Clin Chem 2003, 49:7-18

\section{Pre-publication history}

The pre-publication history for this paper can be accessed here:

http://www.biomedcentral.com/1471-2407/10/693/prepub

\section{doi:10.1186/1471-2407-10-693}

Cite this article as: Chen et al:: Meta-analysis of quantitative diffusionweighted MR imaging in the differential diagnosis of breast lesions. BMC Cancer 2010 10:693.

\section{Submit your next manuscript to BioMed Central and take full advantage of:}

- Convenient online submission

- Thorough peer review

- No space constraints or color figure charges

- Immediate publication on acceptance

- Inclusion in PubMed, CAS, Scopus and Google Scholar

- Research which is freely available for redistribution

Submit your manuscript at www.biomedcentral.com/submit 